\title{
Animationstechnik in der Produktionslogistik
}

\author{
Thomas Kindler
}

Inhalt: Layoutdarstellungen von geplanten Fertigungssystemen haben meist nur wenig Aussagekraft bzgl. der Darstellung von logistischen Prozessen. Nur mit Hilfe einer entsprechenden Darstellung des Projektergebnisses ist es möglich, die zu erfüllenden Systemeigenschaften in anschaulicher Weise auch einem technisch weniger versierten Entscheidungsträger vorzustellen. Der folgende Beitrag soll anhand von Erfahrungen mit der Animationstechnik dazu dienen, Informationsluicken in diesem Bereich zu schließen.

Abstract: Layout representations of planned production systems have usually only few force of expression concerning the representation of logistic processes. Only with the help of an appropriate representation of the project result are possible it to present those to fulfilling system properties in descriptive way also to a technically fewer experienced decision maker. The following contribution is to serve on the basis of experiences with the animation technique to close information gaps within this area.

Im Ergebnis der langjährigen Zusammenarbeit sowohl der Projektlogistik GmbH als auch der Technischen Fachhochschule Wildau mit der RMT Rostocker Maschinenbau und Technologie GmbH \& Co. KG wurden bereits mehrere Forschungs- und Entwicklungsprojekte mit hohem innovativen Inhalt auf dem Gebiet der Umstrukturierung, Rationalisierung und Materialflußplanung sowie Entwicklung von Fertigungssystemen erfolgreich abgeschlossen.
Dieses Unternehmen versteht sich als Zulieferer von Einzelteilen, Baugruppen und Komponenten im Rahmen der Auftragsfertigung im Schiffsmaschinenbau und allgemeinen Maschinenbau. Ein Schwerpunkt der Fertigung liegt in der Herstellung von überlangen Verbindungselementen, wie Zylinderkopfschrauben der Länge 500 - $3000 \mathrm{~mm}$ und einem Kerndurchmesser bis zu $120 \mathrm{~mm}$ sowie den dazugehörigen Muttern, wobei das Unternehmen RMT Rostocker Maschinenbau und Technologie GmbH \& Co. KG derzeit konventionelle Fertigungstechnologien durch moderne Technologien ersetzt. In diesem Zusammenhang wurde an den Logistikdienstleister Projektlogistik $\mathrm{GmbH}$ und die Technische Fachhochschule Wildau der Auftrag erteilt, ein vorhandenes Konzept für ein Fertigungssystem zu optimieren und durch geeignete Software in dreidimensionaler Form den internen prozeß- und materialflußtechnischen Ablauf zu animieren.

Ausgehend von einem manuellen Materialtransport mit entsprechenden Transporthilfsmitteln werden die Maschinen an den einzelnen Arbeitsplätzen durch die $\mathrm{Ar}$ beitskräfte manuell, unterstützt durch technische Hilfsmittel, beschickt und bedient. Damit verbunden entstehen hohe Transport, Lager- und Beschickungszeiten sowie hohe Personalkosten, die keine effiziente Fertigung zulassen.

Im Ergebnis des abgeschlossenen Projektes - Entwicklung eines flexiblen Fertigungssystems für übergroße Verbindungselemente - wurde eine Pilotlösung für den Schiffs- und Schwermaschinenbau entwickelt. In ihr sind

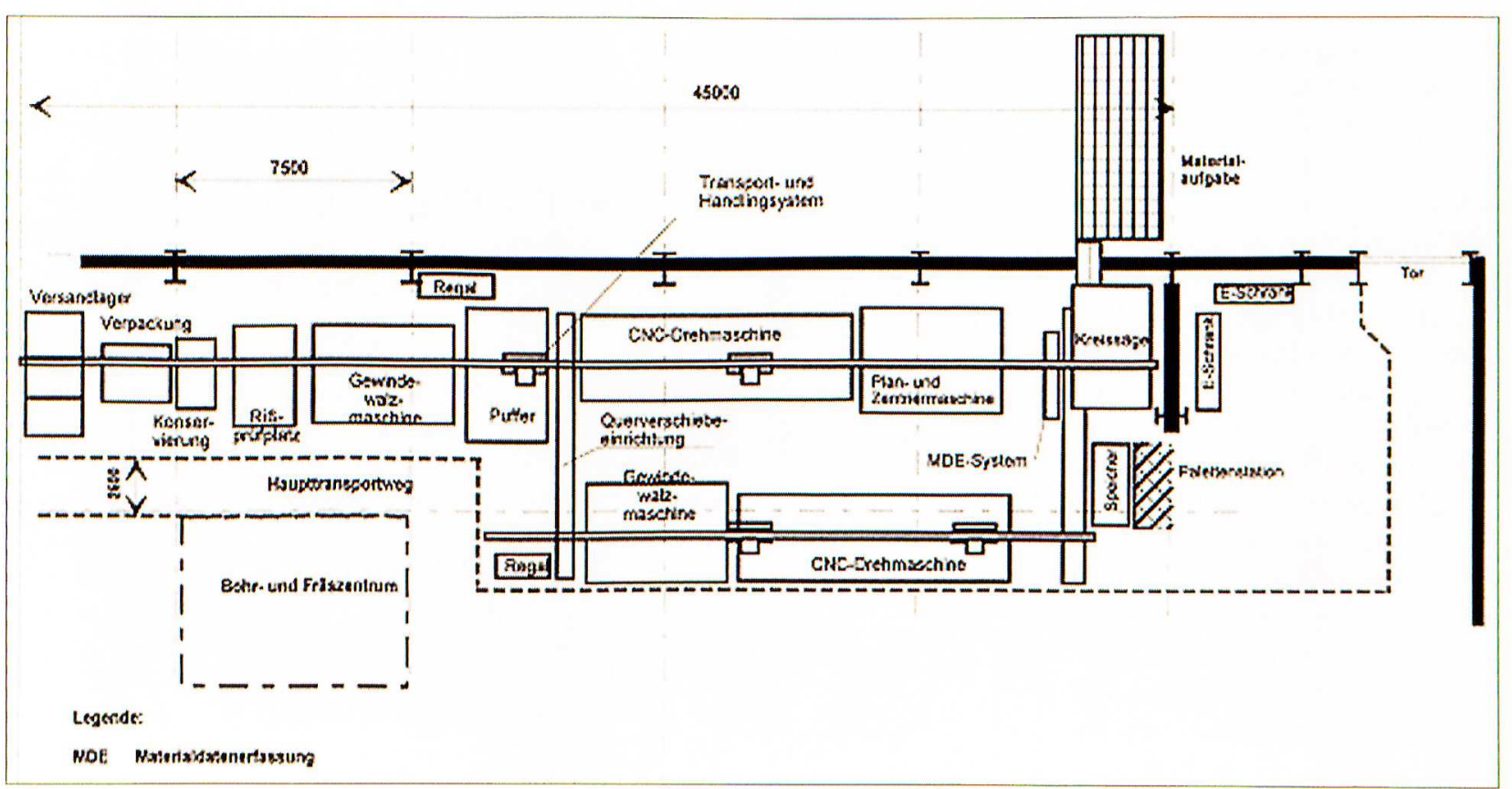

Bild 1: Layout des entwickelten Fertigungssystems [1] 
Logistiklösungen unterschiedlichster Prozesse und der Qualitätssicherung integriert, wodurch ein Konzept für ein neues innovatives Produkt für das Unternehmen RMT entstand. Das innovativ Neue an der konzipierten Fertigungssystemlösung ist, daß trotz der Typenvielfalt der Teile der Gedanke einer Serienfertigung für überlange Verbindungselemente zum Einsatz kommt und somit die Fertigungszeit bzw. Durchlaufzeit entscheidend verkürzt wird. Dabei wurde auf die Umsetzung einer chaotischen Fertigungsauftragsbearbeitung und die Integration eines durchgängigen Qualitätssicherungssystems gesetzt, welches bis zur automatischen Identifizierung und Kennzeichnung der Werkstiicke reicht.

Dieses Produkt in seinem Aufbau und seiner Funktion bis hin zur letzten Detaillösung anschaulich und aussagekräftig einem Interessenten zu präsentieren, ist mit konventionellen Mitteln (2D-Darstellung, 3D-Skizzen) nur unzureichend möglich.

\section{Animation - Anwendung in der Praxis}

Es bestand die Aufgabe, die vorhandenen Darstellungen der Entwicklungslösung durch eine andere Form zu ersetzen, die dem Betrachter ermöglicht, das dargestellte Projektergebnis bzgl. der Integration von Maschinenund Anlagentechnik in einem bestehenden Fertigungsbereich sich räumlich besser vorzustellen, anderseits die geplanten Produktionsprozesse lebendig zu erleben und somit den zur Realisierung erforderlichen Einblick zu erlangen. Mit Hilfe der Animationstechnik, basierend auf der Software zur 3D-Modellierung und Bewegungsbahndefinition von Objekten, wurde eine ansprechende und im Bereich der Produktionstechnik völlig neuartige Konzeptpräsentation erstellt.

Anhand der Darstellung des Fertigungssystems im Bild 1 wird die komplexe und flexibel ausgerichtete Gestaltung deutlich, mit einem zentralen Zwischenlager und einem durchgehenden Materialflußsystem über alle Prozeßstufen.

Im Rahmen der Rationalisierungsmaßnahme im Unternehmen RMT Rostocker Maschinenbau und Technologie GmbH \& Co. KG standen wir vor der Aufgabe, die technischen und technologischen Prozesse bei der Schraubenherstellung und deren parallel im System ablaufenden Prozeßschritte zu beachten. Diese technischen Prozesse werden durch aneinander gekoppelte Arbeitsschritte, speziell bei den Umform- und Abtrennverfahren, charakterisiert, die nur in einer bestimmten Reihenfolge ablaufen können und genau definierte Bearbeitungsparameter erfordern. Nur mit Hilfe einer entsprechenden Darstellung der Projektergebnisse ist es möglich, die zu erfuillenden Systemeigenschaften in anschaulicher Weise auch ei- nem technisch weniger versierten Entscheidungsträger zu präsentieren.

Während der Feinplanung innerhalb des Projektes wurden dessen Ergebnisse als Layoute und Materialflußschemata festgehalten, ohne dabei wirklich die Dynamik und Flexibilität der entwickelten Fertigungsanlage darstellen zu können (s. Bild 2).

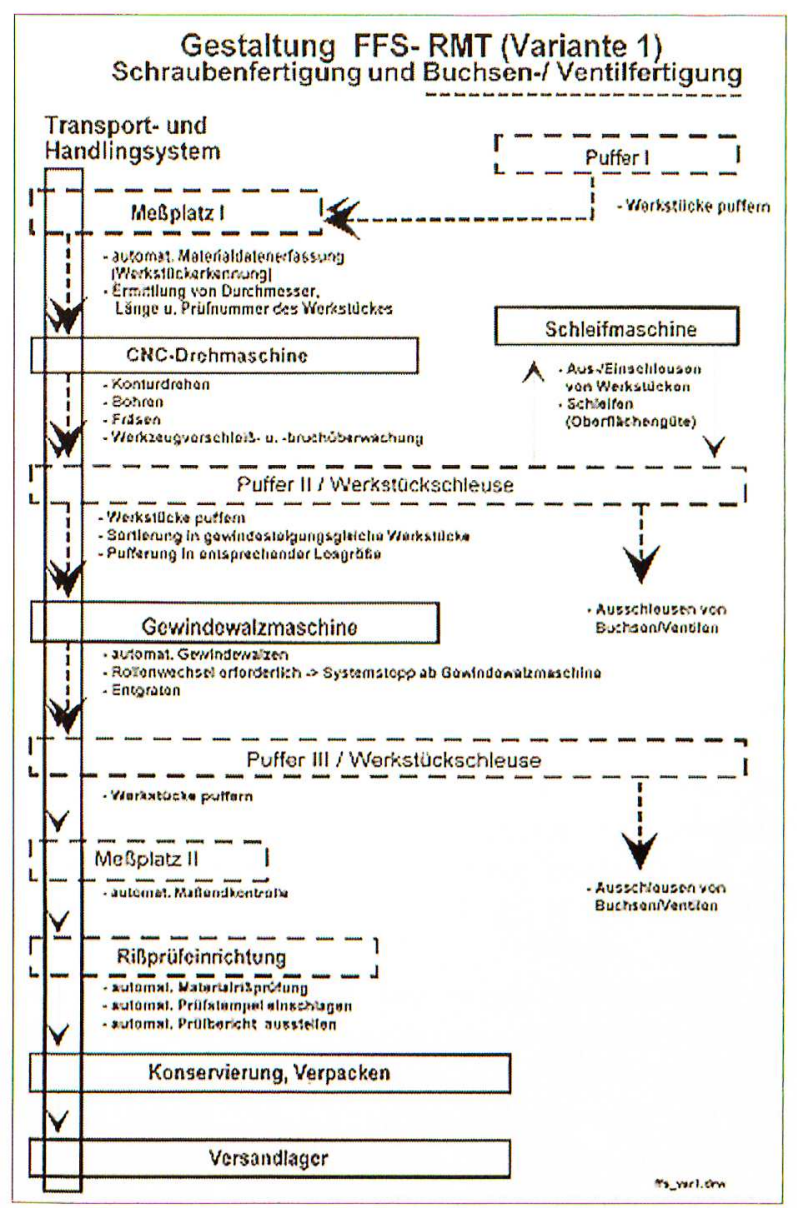

Bild 2: Materialflußschema für das Fertigungssystem

Oft sind selbst so einfache Handlingsaufgaben, wie das Drehen des Werkstuickes um $180^{\circ}$ über einer Maschine mittels Transportgreifer, nur unzureichend als schematische Abbildung im Layout darstellbar (s. Bild 3). Werden dann noch Anforderungen an die räumliche

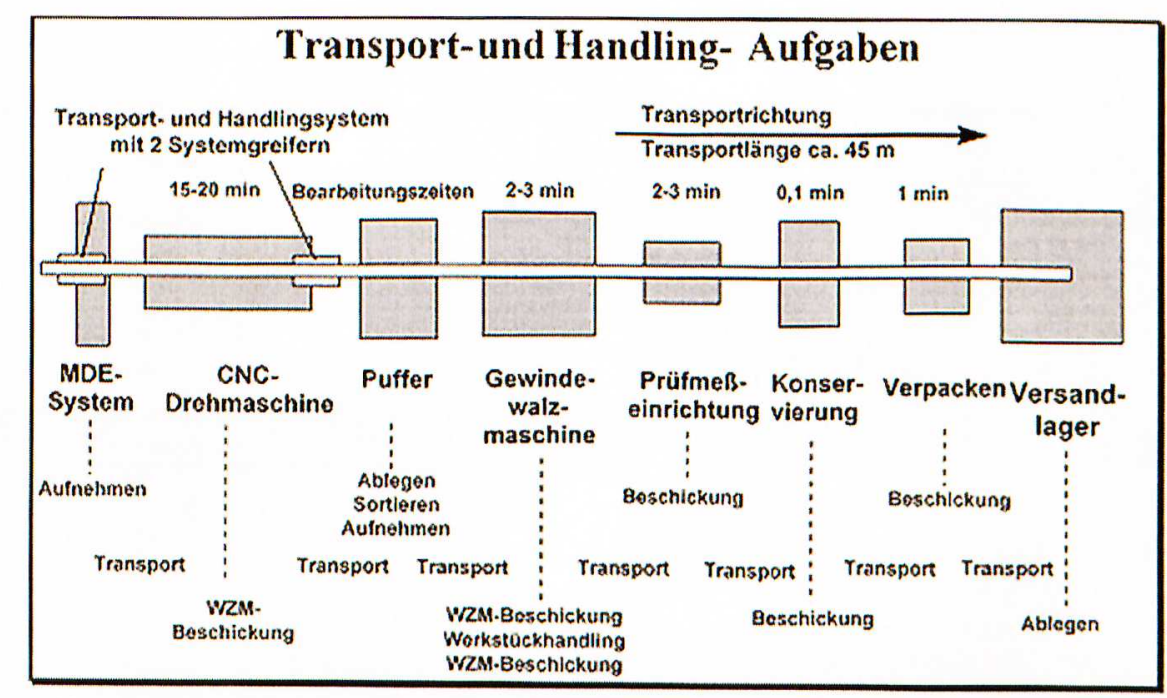

Bild 3: Transport- und Handlingsaufgaben im Fertigungssystem [1] 
Anordnung und Zugänglichkeit der Maschinen gesetzt, können nur die Informationen entnommen werden, die eine 2-dimensionale Layoutansicht zuläßt. Maximal eine Höhenschnittdarstellung kann zum Teil Aufschluß über etwaige Kollisionszustände oder Einschränkungen geben.

Die folgende animationstechnisch erstellte Darstellung des o. g. Fertigungssystems (s. Bild 4) beinhaltet alle wesentlichen Systemeigenschaften, die im Ergebnis der Entwicklungsarbeit entstanden sind. Dies beläuft sich auf die Darstellung der räumlichen Anordnung der einzelnen Bearbeitungsmaschinen, der Puffertechnik und Handlingstechnik sowie der einzelnen technologischen und logistischen Prozesse im System.

Als Darstellungsmittel des Ergebnisses der Projektarbeit wurden die bereits aufgefuihrten Layoute und Materialflußschemata als Grundlage gewählt, wobei mehrere Ausbaustufen zu beriicksichtigen waren.

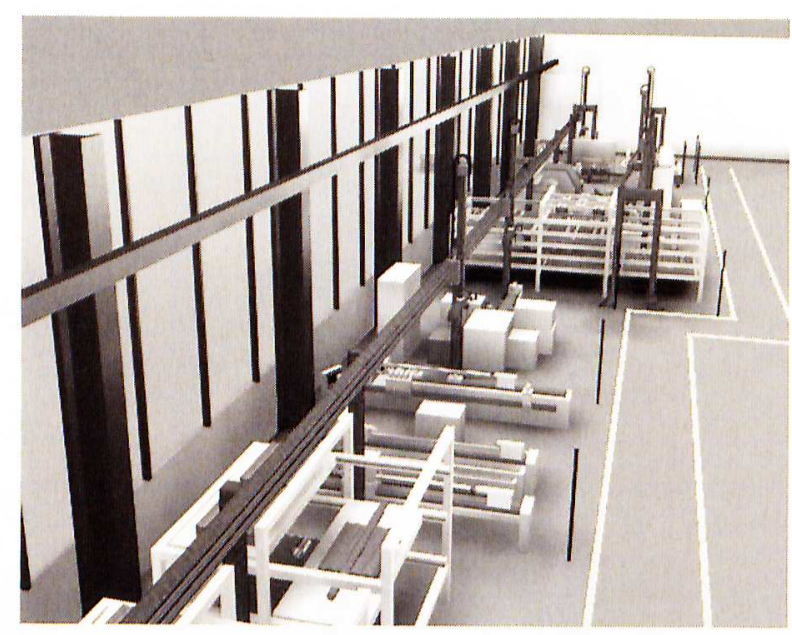

Bild 4: Szene aus Fertigungssystemanimation [2]

Besonderes Augenmerk wurde auf das Zusammenspiel zwischen der Handlingstechnik mit den Bearbeitungsmaschinen und den Puffereinrichtungen gesetzt (s. Bild 5).

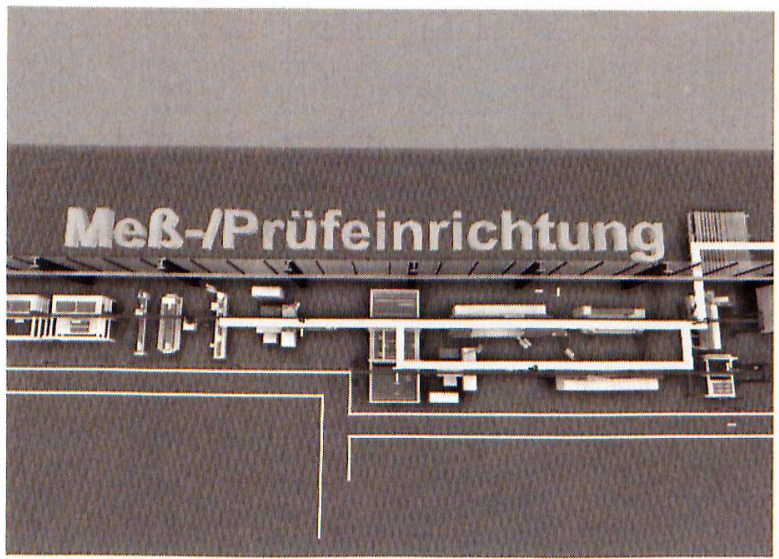

Bild 5: Darstellung des Materialflusses [2]

Schon bei der Erstellung der Szenen konnte durch die dabei unterschiedlich definierten perspektivischen Ansichten eines Systembereiches eine Kollision von Objekten visuell ausgeschlossen werden.
Der Aufbau der Animation setzt sich aus folgenden Inhaltsschwerpunkten zusammen:

> Intro - Logo des Unternehmens und Titel,

> Effektvolle Darstellung des flexiblen Fertigungssystems (FFS) durch Nebel und Volumenlicht

- Kamerafahrt über FFS bzgl. Gesamteindruck

> Darstellung des Materialflusses von Dehnbolzen und Buchsen innerhalb des FFS

$>$ Darstellung der Arbeitsgänge zur Herstellung eines Dehnbolzens

$>$ Zuschnitt in der Kreissäge

$>$ Transport zur Plan-/Zentriermaschine mit $90^{\circ}$ Drehung, Plandrehen/Zentrierbohren

> Transport zur CNC-Drehmaschine, Konturdrehen 1

$>180^{\circ}$-Drehung des Werkstïckes, Konturdrehen 2

> Transport zur Profilwalzmaschine, Gewindewalzen 1. Gewinde

$>180^{\circ}$-Drehung des Werkstiickes, Gewindewalzen 2. Gewinde

$>$ Transport zum Zwischenlager, Ablegen auf Palette

$>$ Aufnahme eines 2. Werkstückes, Transport zur Profilwalzmaschine

$>$ Gewindewalzen 3. Gewinde

> Transport zur Meß-/Prüfeinrichtung mit $90^{\circ}$ Drehung,

> Maßhaltigkeitskontrolle, Materialrißprüfung, Kennzeichnung des Werkstuickes

> Transport zur Konservierungsmaschine, Konservierung mittels Ölnebel

> Transport zur Verpackungsmaschine, Verpacken mit Kunststoffnetz

> Transport zum Versandlager, Ablegen auf Palette $>$ Abspann

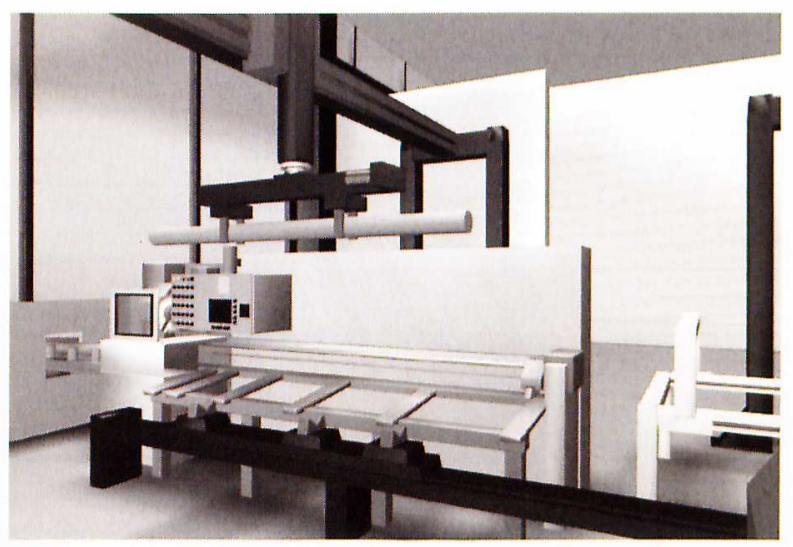

Bild 6: Szene aus Animation - Entnahme Rohling am Zuschnitt [2]

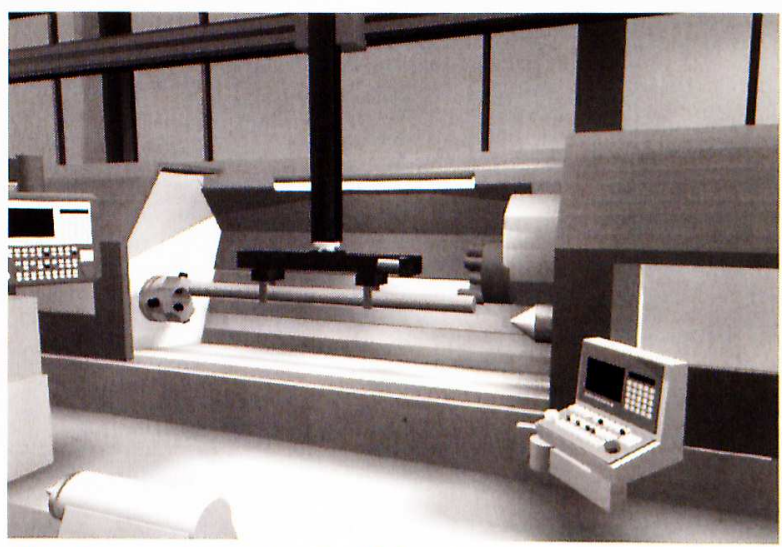

Bild 7: Szene aus Animation - Beschickung der CNC-Drehmaschine [2] 


\section{Vom Layout zur Animation}

Aus einer ungefähren Vorstellung, wie die Besonderheiten und Aktionen in der Animationsszene dargestellt werden sollen, und daraus einen zusammenhängenden Film zu erstellen, ist nur mit Hilfe eines Drehbuches möglich. In diesem Script müssen in Abhängigkeit von der Zeit alle Objekte, deren Bewegungen und Kameraeinstellungen, definiert sein (s. Bild 8). fotorealistischen Darstellung angepaßt.

Erfolgte die Modellierung aller zur Animation erforderlichen Objekte, werden die im Aufnahmemodus in dem im Script vorgegebenen Zeitbereich bzgl. der Position bzw. Geometrie verändert. Entsprechende Kameraeinstellungen und -bewegungen werden ebenfalls nach dieser Methode in der Animation definiert.

Licht und Schatten oder sonstige atmosphärische Effekte geben einer Animation erst den besonderen Aus-

\begin{tabular}{|c|c|c|c|c|c|c|}
\hline Animation FSS & & & ip1 Greifer 2 & & & \\
\hline Zsilt (s)] & Akdion & Kamaral & Kamera 2 & $\mathrm{BEO}$ & $6 \mathrm{EO} 2$ & $\mathrm{BEO}$ \\
\hline $0-5$ & Vetchen won Gefer 2 & & & L.o.twogen 2 & Geiter 2 & \\
\hline 5.10 & Neronkon won Greiter 2 & & & Grofer? & & \\
\hline 10.12 & SchigQsen der Groiffningar & & & Grelfinger 5 urdo & Geifingor 7 und 8 & \\
\hline $12 \cdot 13$ & Losen der Elnspornung & & & Scorniutte: I & Zentrlarspiza I & \\
\hline 13.15 & 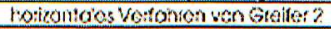 & & & 10uffoggen? & Goifer? & Ronirg 70 \\
\hline $15-20$ & Haben von Gififer 2 & & & Gerig: 2 & Ronirgalo & \\
\hline 20.35 & Varfohran zur 'scizm? & & Schwenk tind Powegung & loukvegen? & Geter 2 & Ronirg $7 \mathrm{~b}$ \\
\hline 35.00 & Nesonksan wen Gloifor 2 & & & Groife: 2 & Rolaling $7 b$ & \\
\hline$\$ 02.42$ & Enspomanne won Rorilag 70 & & & Zeninerung I & & \\
\hline 42.43 & Olfnencer Greilfinger & & & Geotrmet Buads & Gouffinger 7 uncia & \\
\hline$\Delta 3 . \Delta \Delta$ & Habenvon Cagifgr ? & & & Groifo: 2 & & \\
\hline 46.130 & WalzproxoBs & & & Walzo y & WWalzo? & Fohiling 70 \\
\hline 130.133 & Aexonken von Gleiter? & & & Gealle: ? & & \\
\hline $133-135$ & Seniszon der Groiffinger & & & Gosfrowe: 5unss & Groffrose 7 ures 9 & \\
\hline $136-137$ & L.csan der ilnsponnurg & & & Zentrenga & & \\
\hline $137-142$ & Kebenvon Groiter? & & & Greite! ? & Hobzeuci $7 a$ & \\
\hline $142-147$ & 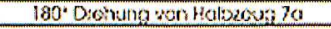 & & & Gtolio: 2 & Habroun $7 a$ & \\
\hline $147-169$ & Follzonto as Vertohren won Greliar 2 & & & Louhwogen 2 & Greffe: 2 & Halozeug 70 \\
\hline $149-154$ & Absanken ven Greifer 2 & & & Gellet 2 & & \\
\hline 154.150 & Eirsoxarruma von Hicibzeung 70 & & & Zenltionum ! & & \\
\hline $156 \cdot 157$ & Offinen dar Gralfiningar & & & Qoffingo: 5 uns & Glalfrnge: 7 und 8 & \\
\hline $157-160$ & Habenvon Grefer 2 & & & Gralle: 2 & & \\
\hline
\end{tabular}

Bild 8: Sript zur Animationserstellung [2]

Ist die Visualisierung einer großen Anzahl von Objekten angedacht, so ist die Aufstellung eines vergleichenden Zeitablaufplanes für alle zu bewegenden Objekte erforderlich, somit wird eine unbeabsichtigte Kollision von Objekten schon von vornherein ausgeschlossen (s. Bild 9). druck. Dazu werden die unterschiedlichen Lichtquellen (Umgebungslicht, Punktlicht, Spot) an bestimmten Punkten im Raum generiert und durch einen vereinfachten Renderingmodus (Verfahren zur Oberflächenberechnung und -darstellung) auf deren Wirkung geprüft. Sind atmosphärische Effekte (Volumenlicht, Ne-

Animation FSS

Script Greifer 2

Schleife

\begin{tabular}{|c|c|}
\hline 2 git (s) & Asklion \\
\hline $0-5$ & Vertohen won Grefer 2 \\
\hline 5.10 & Natonken ven Greiler? \\
\hline 10.12 & Schiogen der Graiffingar \\
\hline $12-13$ & Losan de Elnspornung \\
\hline 13.15 & 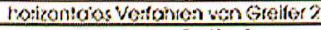 \\
\hline $15-20$ & Haban von Gelfer 2 \\
\hline 20.35 & Verfolken zur woram2 \\
\hline 35.40 & Abonken ven Groifor 2 \\
\hline 00.42 & Elntpanzung won Rorilag 70 \\
\hline 12.43 & Olfnen cer Grailfinger \\
\hline 43.46 & Haben von Crailer ? \\
\hline $46-130$ & Walzproxoß31 \\
\hline 130.133 & Seonkenven Greiter 2 \\
\hline 133.135 & Schig/enclar Groiffinger \\
\hline $135-137$ & L.csan dot Elmsponnurg \\
\hline 137.142 & Hothan won exofer 2 \\
\hline $142-147$ & 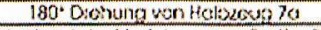 \\
\hline 147.1 .48 & Forizonto'as Vertohian ven Grelier 2 \\
\hline $199-154$ & Arsenken von Greiter? \\
\hline $184 \cdot 153$ & Eirsex:mrema; von Habzoun $7 a$ \\
\hline 156.157 & Offinzn ckar Grolffingar \\
\hline $157-160$ & Haben von Gelier 2 \\
\hline
\end{tabular}

\begin{tabular}{|c|c|}
\hline Kamero 2 & \\
\hline & \\
\hline & \\
\hline & \\
\hline & \\
\hline & \\
\hline & \\
\hline & \\
\hline & \\
\hline & \\
\hline
\end{tabular}

\begin{tabular}{|c|}
\hline BEO \\
\hline Lostwogen 2 \\
\hline Grofor 2 \\
\hline Graiffingor 5 undo \\
\hline Sconniutter I \\
\hline loufworgen? \\
\hline Crengs 2 \\
\hline 1.oufvicgen? \\
\hline Gioile: 2 \\
\hline Zanitielung 1 \\
\hline Gretrones Bundo \\
\hline Giolify: $z$ \\
\hline Wolzo 1 \\
\hline Greiler? \\
\hline Goiffrxa: 5 unas \\
\hline 2entranum 1 \\
\hline Greilear? \\
\hline Gtoifo: ? \\
\hline Loufivgen 2 \\
\hline Greiter? \\
\hline Zentrienum i \\
\hline$\frac{\text { Goiffrogen 5und } 6}{\text { Gralfer } 2}$ \\
\hline
\end{tabular}

\begin{tabular}{|c|c|}
\hline $\mathrm{BEO} 2$ & $98{ }_{3}$ \\
\hline \multicolumn{2}{|l|}{ Greter 2} \\
\hline \multicolumn{2}{|l|}{ Cigifinger 7 und 8} \\
\hline \multicolumn{2}{|l|}{ Zentrlarspo.tze I } \\
\hline Goter 2 & Ronirgy 70 \\
\hline \multicolumn{2}{|l|}{ Ronirgalo } \\
\hline Geter? & Rohing $7 b$ \\
\hline \multicolumn{2}{|l|}{ Rohiling; $7 \mathrm{~b}$} \\
\hline \multicolumn{2}{|l|}{ Getrirsed 7 urci 8} \\
\hline Wolzon & Fohling 70 \\
\hline \multicolumn{2}{|l|}{ Greifroger 7 und 8} \\
\hline \multicolumn{2}{|l|}{ Habreug 70} \\
\hline \multicolumn{2}{|l|}{ Habroug 70} \\
\hline Grofer 2 & Holbzeng 70 \\
\hline & \\
\hline Glaffrnger 7 und 8 & \\
\hline
\end{tabular}

Bild 9: Zeitablaufplan für Animationsobjekte [2]

Nach der Scripterstellung erfolgt die Objektmodellierung, wobei das Script Anforderungen an die zu modellierenden Objektgruppen vorgibt, da alle zu bewegenden Teile möglichst mit den im Script definierten Objektnamen und Eigenschaften übereinstimmen müssen (s. Bild 10).

Im nächsten Arbeitsschritt werden mittels einer umfangreichen Auswahl an Materialoberflächen und Texturen die als Volumenmodelle erstellten Objekte ihren in der Realität existierenden Originalen bzgl. einer möglichst bel) in bestimmten Szenen vorgesehen, werden diese durch spezielle Einstellungen im Menübereich der Umgebungsparameter erzeugt.

Im nachhinein können innerhalb der Animationserstellung in einer speziellen Spuransicht, die sämtliche Modifikationsparameter für Objekte, Klang, Umgebung und Szenenmaterialien enthält, noch Änderungen vorgenommen werden. So ist z. B. die Drehzahl des Spannfutters der CNC-Drehmaschine durch Verändern des Drehwinkels im unveränderten Zeitbereich 
modifizierbar. In dieser Spuransicht kann nachträglich die Szene durch Einfügen einer Sounddatei vertont werden, so daß eine Animation mit Klangeffekten entsteht.

Das Rendering - Routine zur Oberflächenberechnung und -darstellung - steht nach Abschluß aller vorbereitenden Arbeitsschritte als eine der wichtigsten Erstellungsroutine an. Die berechneten Einzelbilder werden mittels Videoschnittsoftware zu einem Videoclip verarbeitet. Dabei wird der Animation durch Vertonen der letzte Schliff gegeben und als Video auf einen geeigneten Medienträger (S-VHS-Videoband) abgespeichert.

\section{Ausblick}

Aufgrund der detailgetreuen Darstellung des Fertigungssystems und der darin ablaufenden logistischen Prozesse wurde mit dieser Animation ein Präsentationsmittel für die RMT Rostocker Maschinenbau und Technologie GmbH \& Co. KG geschaffen, das besonders bei Firmenpräsentationen auf Messen und Ausstellungen von diesem Unternehmen bereits intensiv genutzt wurde. Diese Animation wurde weiterhin als Bestandteil fuir die Erstellung von Internet-Seiten und einer UnternehmensPräsentations-CD verwendet.

Als sehr wesentlich erwies sich jedoch der Schulungseffekt für die Werker. In jeder Phase des Prozesses konnten sie sich die Veränderungen bzw. Neuerungen wirklichkeitsgetreu darstellen lassen. Dies führte zur inhaltlichen Präzisierung und auch zur Identifikation mit der neuen technologischen Prozeßdarstellung.

Die ausgezeichneten Erfahrungen, die das Unternehmen RMT mit dem neuen Präsentationsmittel Animation gewonnen hat, gaben den Anlaß, alle weiteren Projektergebnisse animationstechnisch erstellen zu lassen und die generelle Einführung neuer innovativer Konzepte mit einer derartigen räumlichen Animation abschließen zu lassen.

Weiterhin gibt es Überlegungen, die Darstellungsmöglichkeiten im Bereich Virtual Reality und Simulation bei der Projektierung und Entwicklung von technischen Systemen zu integrieren, wobei die drei folgenden Ansätze gegeben sind.

Eine Begehung des FFS mittels 3D-Brille unter Verwendung einer geeigneten Virtual- Reality- Software zu realisieren, ist eine Möglichkeit der interaktiven Betrachtung eines virtuellen Raumes. Dabei können sämtliche Fertigungsprozesse dargestellt werden, ohne aber Einfluß auf diese zu besitzen.

Als weitere Möglichkeit ist der Einsatz eines Datenhandschuhes als Grundlage für die virtuelle Fabrik- und Logistikplanung zu nennen. Hierbei wird dem Fabrikplaner die Möglichkeit gegeben, mittels bereits genanntem Datenhandschuh die Maschinen in ihrer Geometrie und räumlichen Anordnung zu verändern, wobei sich dieser selbst virtuell mitten im System befindet.
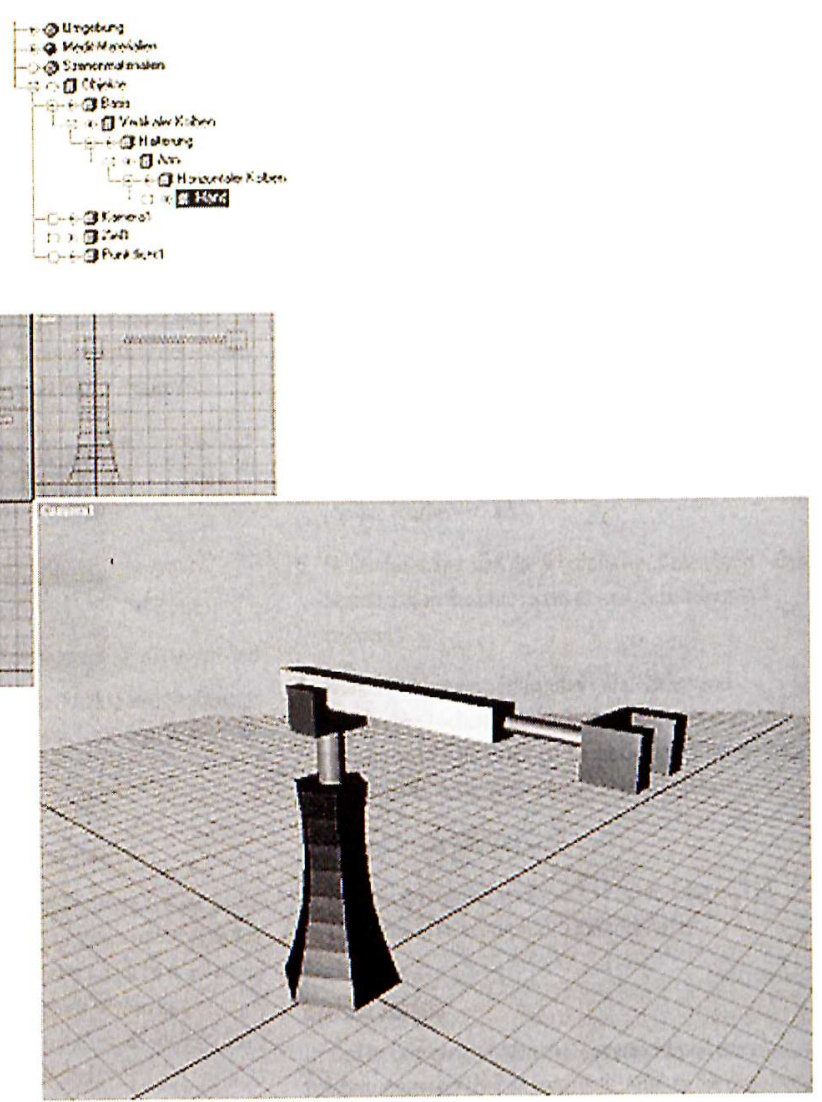

Bild 10: Beispiel der Objektmodellierung [3]

Der 3. vorstellbare Ansatz zur Verbesserung der Planungsmethoden und -hilfsmittel ist die 3D-Darstellung des Systems und die gleichzeitig vorhandene Option, die Eingangsparameter zur Simulation des Fertigungsprozesses jederzeit verändern zu können.

\section{Literatur}

[1] Tscherner, P., Kindler, Th., Neuhaus, Ch.: Entwicklung eines flexiblen Fertigungssystems für übergroße Verbindungselemente mit integrierter Qualitätssicherung in der RMT Rostocker Maschinenbau und Technologie GmbH \& Co. KG - Projektdokumentation des Fraunhofer Anwendungszentrums für Verkehrs- und Entsorgungslogistik, 1997 und Prof. B. Hentschel, Tscherner, P., Kindler, Th., Saffian, K.: Flexibles Materialflußkonzept für ein Fertigungssystem, Jahrbuch der Logistik, 1998

[2] Kindler, Th.: Animation - Flexibles Fertigungssystem, Projektunterlagen der RMT Rostocker Maschinenbau und Technologie GmbH \& Co. KG, 1998

[3] Handbuch - 3D Studio MAX

\section{Autor}

Dipl.-Ing. Thomas Kindler

Technische Fachhochschule Wildau

Produktionslogistik

Bahnhofstraße

15745 Wildau

Tel. (0 33 75) 508-930 